\title{
Compte-rendu des \\ Journées 'Machines hydrauliques, Cavitation' 2017 \\ ENSAM, Campus de Paris \\ 8 et 9 Novembre 2017
}
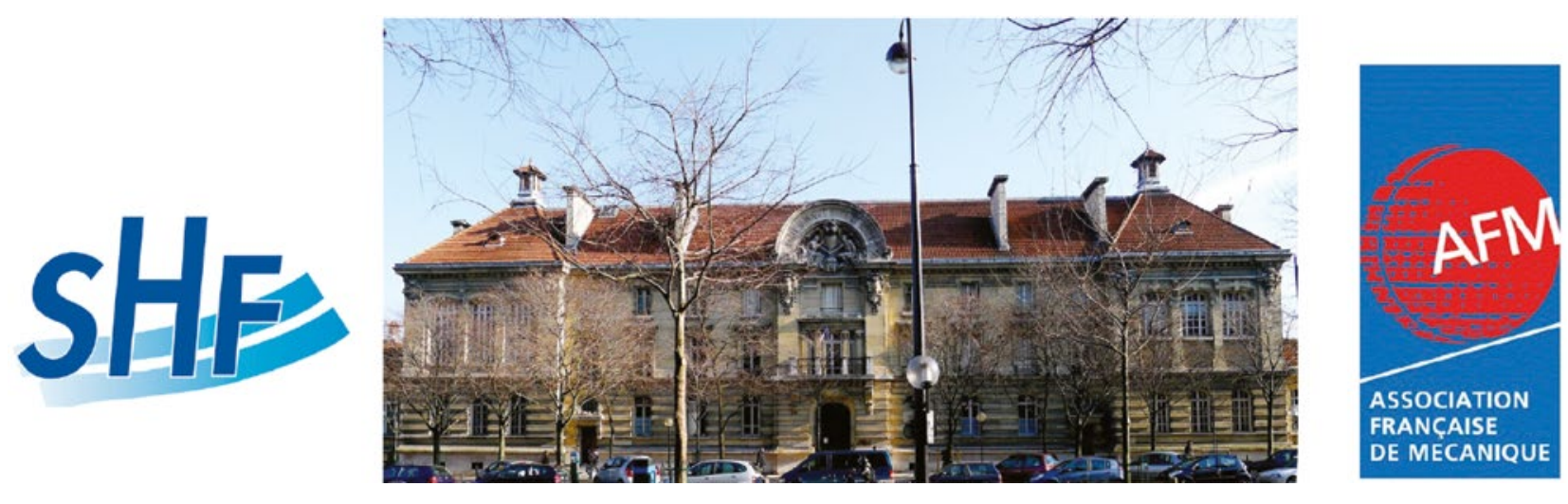

Antoine Dazin ${ }^{1}$, Sofiane Khelladi², Antoine Archer ${ }^{3}$

1 ENSAM, Lille

2 ENSAM, Paris

3 EDF, Chatou

Les journées 'Machines hydrauliques et cavitation' se sont tenues les 8 et 9 Novembre 2017 dans les locaux du Campus ENSAM de Paris.

Cette manifestation a été organisée avec le soutien de la SHF, de l'AFM et de l'ENSAM. Elle a réuni 39 participants de 8 nationalités différentes. La majorité des présentations ont été données en anglais. La répartition était relativement équilibrée entre monde académique (17 participants) et industriel (22 participants : fabricants de machines hydrauliques, installateurs, motoristes, exploitants de réseaux hydrauliques et de barrages, bureaux d'études, centres techniques ou de recherche).

La fourniture d'un résumé et/ou une inscription par mél étaient seules requises pour la participation à ces journées. Ces journées ont été parfaitement organisées et prises en charge par le Campus ENSAM de Paris, avec le soutien de la Société Hydrotechnique de France. En fin de conférence, un encouragement à publier dans la Houille Blanche a été exprimé. Enfin, une visite des installations d'essais du Laboratoire DynFluid dans les locaux de l'ENSAM (machines hydrauliques et aérauliques) a été organisée en clôture des journées.

$\mathrm{Au}$ total, 25 présentations ont été proposées lors de ces deux journées, réparties en 7 sessions successives.

Le thème général portait sur les machines hydrauliques et la cavitation et les présentations ont porté aussi bien :

— sur des retours d'expériences industriels à l'échelle de la machine ou du système

- que sur des travaux plus amont portant sur la modélisation ou la compréhension des mécanismes physiques associés aux écoulements internes aux machines hydrauliques et/ ou à la cavitation.

Plus précisément, sur la cavitation, les présentations ont particulièrement mis l'accent sur :

- La compréhension, par des approches expérimentales, des mécanismes physiques mis en jeu à petite échelle [1], à bas nombre de Reynolds [2] ou dans les tourbillons marginaux [3].

- Des travaux de modélisation numérique de ce type d'écoulement à l'échelle d'une maquette de type foil [4] jusqu'à des machines industrielles [5-7].

- L'érosion de cavitation, du point de vue de son impact sur des turbines Kaplan [8], de sa métrologie [9] ou de l'étude des matériaux les plus performants pour retarder ses effets négatifs [10]

- Le dessin et de la performance des inducteurs [11-12].

- La détection de l'apparition de la cavitation ou du collapse par des méthodes acoustiques [13-14].

Dans le domaine des machines hydrauliques ou aérauliques, les travaux présentés lors de ces journées ont porté sur de l'analyse de la physique, à l'échelle du composant [15], ou de la machine lorsqu'elle présente des régimes de fonctionnement instable [16], mais aussi sur de la modélisation fonctionnelle des pompes [17] ou sur des dessins originaux pour des applications particulières dans le domaine de l'industrie pétrolière [18] ou de la production d'énergie [19].

On peut également noter les travaux portant sur la modélisation système, par de la modélisation CFD [20-21] ou par des approches monodimensionnelles pour la prise en compte de certains effets transitoires [22] ou de la dynamique des structures de cavitation [23].

Enfin, citons finalement, deux retours d'expérience rapportant plusieurs décennies de travaux sur, d'une part, l'utilisation de codes de calculs industriels pour le dimensionnement et l'optimisation d'installations hydro-électriques [24] et d'autre part sur le design et l'exploitation de boucles expérimentales pour l'étude de la cavitation [25].

L'ensemble des présentations a ainsi offert un aperçu riche et très varié des travaux en cours dans le domaine des turbomachines hydrauliques. Deux points marquants qui ressortent de ces journées peuvent être notés :

- le premier concerne l'utilisation de plus en plus courante de modèles numériques de simulation d'écoulements à 
l'échelle locale pour le traitement de ces problèmes, y compris à l'échelle de machines industrielles. Cela traduit, d'une part, l'augmentation de la puissance de calculs à disposition des laboratoires et des entreprises, mais aussi, d'autre part, une maîtrise de plus en plus grande de ces outils, de leurs possibilités et de leurs limites.

- Le second concerne le besoin de validation des résultats de ces modélisations numériques et l'amélioration des modèles, qui nécessitent des expérimentations de plus en plus fines et le développement de métrologie adaptée aux échelles pertinentes en temps et en espace.

La qualité des échanges et la bonne participation à ces journées devrait permettre de reconduire leur organisation d'ici deux ans, dans un lieu qui reste à déterminer.

\section{Liste des présentations}

[1] Cavitation Dynamics at Sub-Millimeter Scale- K. Long, M. Dular, O. Coutier-Delgosha- ENSAM - LML, France / Université de Lujbana, Slovénie

[2] Etude Expérimentale de la Cavitation à Bas Nombre de Reynolds- K. Croci, F. Ravelet, J-C. Robinet, A. DanlosENSAM-DynFluid/CNAM, France

[3] Role of Gas Content on Tip Vortex Cavitation, A. Amini, M. Farhat, EPFL, Suisse

[4] Numerical investigation of the flow around a NACA0015 near cavitation inception - J.Decaix, G. Balarac, C. Munch - Univ. of Applied Sciences and Arts, Suisse/Universite Grenoble Alpes-LEGI, France

[5] Prévision de l'intensité de cavitation sur une pompe centrifuge à partir de simulations numériques avec Code-Saturne - Premiers résultats sur la pompe SHF - Ch. Leclercq, R. Fortes-Patella, A. Archer, F. Cerru - Université Grenoble Alpes-LEGI/EDF, France

[6] Numerical Methodology to Predict and Analyze Cavitating Flow in a Kaplan Turbine- F. Turi, R. Fortes-Patella- Université Grenoble Alpes-LEGI, France

[7] 3D steady and transient cavitation flow on very low specific speed centrifugal pump- C. Leroy, D. Fedala, G. Boitel, N. Myon - SULZER Ensival Moret, France

[8] Cavitation of Francis Turbine HPP Piva and applied methods for reducing negative impact- Z. Giljen, M. Nedeljković, Montenegro Electric Company/University of Belgrade

[9] Measurement of Cavitation Aggressiveness Using PVDF Sensors and Comparison with Numerical Results- J.-B. Carrat, J.-P. Franc, R. Fortes-Patella, Université Grenoble Alpes-LEGI

[10] Experimental approaches to mitigate cavitation erosion deleterious effect in hydraulic turbine runners, L. Ton-That Hydro-Quebec, Canada
[11] Experimental assessment of the influence of the hub shape on the performances of an axial inducer operating under cavitation regime - R. Paridaens, M. Solis, C. Dupuy, S. Khelladi, F. Bakir, ENSAM-DynFluid, France

[12] Experimental Investigation of the Coupled Effect of Degassing and Cavitation on the Performances of an Inducer- P. Tomov, R. Pariaens, F. Bakir, S. Khelladi, L. Pora, SNECMA/ ENSAM-DynFluid, France

[13] Detection of Cavitation Onset in Hydraulic Systems G. Pavic, F. Chevillotte- INSALyon/Matelys Research, France

[14] Cavitation bubble collapse detection by acoustic emission - M. Ylonen, P. Saarenrinne, J. Miettinen, J.-P. Franc, M. Fivel, V. Kokko, Tampere University of Technology, Finland/Universite Grenoble Alpes-LEGI/Universite Grenoble Alpes-SIMaP, France

[15] The Effect of the Reduced Frequency on Oscillating Flexible Hydrofoils- S. Hoerner, S. Abbaszadeh, Th. Maitre, C.-T. Weber, R. Leidhold, D. Thevenin, University Magdeburg, Allemagne/Universite Grenoble Alpes-LEGI, France

[16] Stability analysis of rotating stall in a wide vaneless diffuserY. G. Heng, A. Dazin, M. N.Ouarzazi- LML - ENSAM / Université Lille, France

[17] Modélisation unidimensionnelle du comportement d'une pompe rotodynamique en fonctionnement normal et accidentelL. Matteo, A. Dazin- CEA, LML / ENSAM, France

[18] Enhance Heavy Oil Transportation by New optimized jets pumps- M. Asuaje, R. Noguera- Universidad Simon Bolivar, Venezuela/ENSAM-DynFluid, France

[19] Design et simulation numérique 2D de turbines à double flux pour une implantation dans le tunnel sous la MancheTh. Delecour, A.-C. Bayeul-Laine, O. Coutier-Delgosha, A. Dazin, ENSAM-LML, France

[20] Evaluation du risque d'un mauvais fonctionnement d'une station de pompage à partir d'une simulation CFD- G. Guibu Pereira, J. Wertel, M. Dufresne, S. Isel, J. Vazquez- ICUBE/3D EAU, France

[21] Analyse numérique des pertes dans un conduit d'écluse fluviale - A. Achhoud, A.-C. Bayeul-Laine, A. Dazin - LML - ENSAM

[22] Modélisation du risque de coup de clapet appliquée à une grosse installation de refoulement d'eau potable - M. Lafforgue - Suez Consulting, France

[23] Mass flow gain effect of a cavitating draft tube vortex P. K. Doerfler - Hydro adviser LLC, Suisse

[24] A Review of CFD studies Applied to hydraulic turbines generation systems, M. Asuaje, R. Noguera, Universidad Simon Bolivar, Venezuela/ENSAM-DynFluid, France

[25] Retour d'expérience sur la conception et l'exploitation d'installations de cavitation. Y Lecoffre, Hydeo France 


\section{SHE}
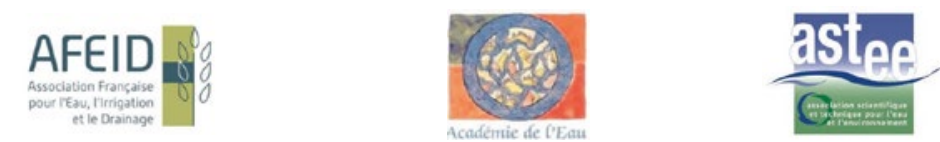

Congrès ASTEE, Marseille - Jeudi 7 juin 2018

Side-event du groupe de travail inter-associatif « Tensions sur l'Eau »

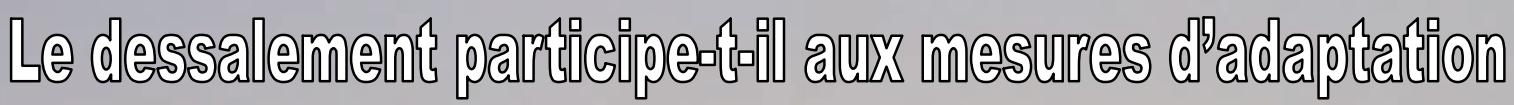
au changement chlimatidgue ?

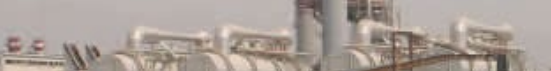

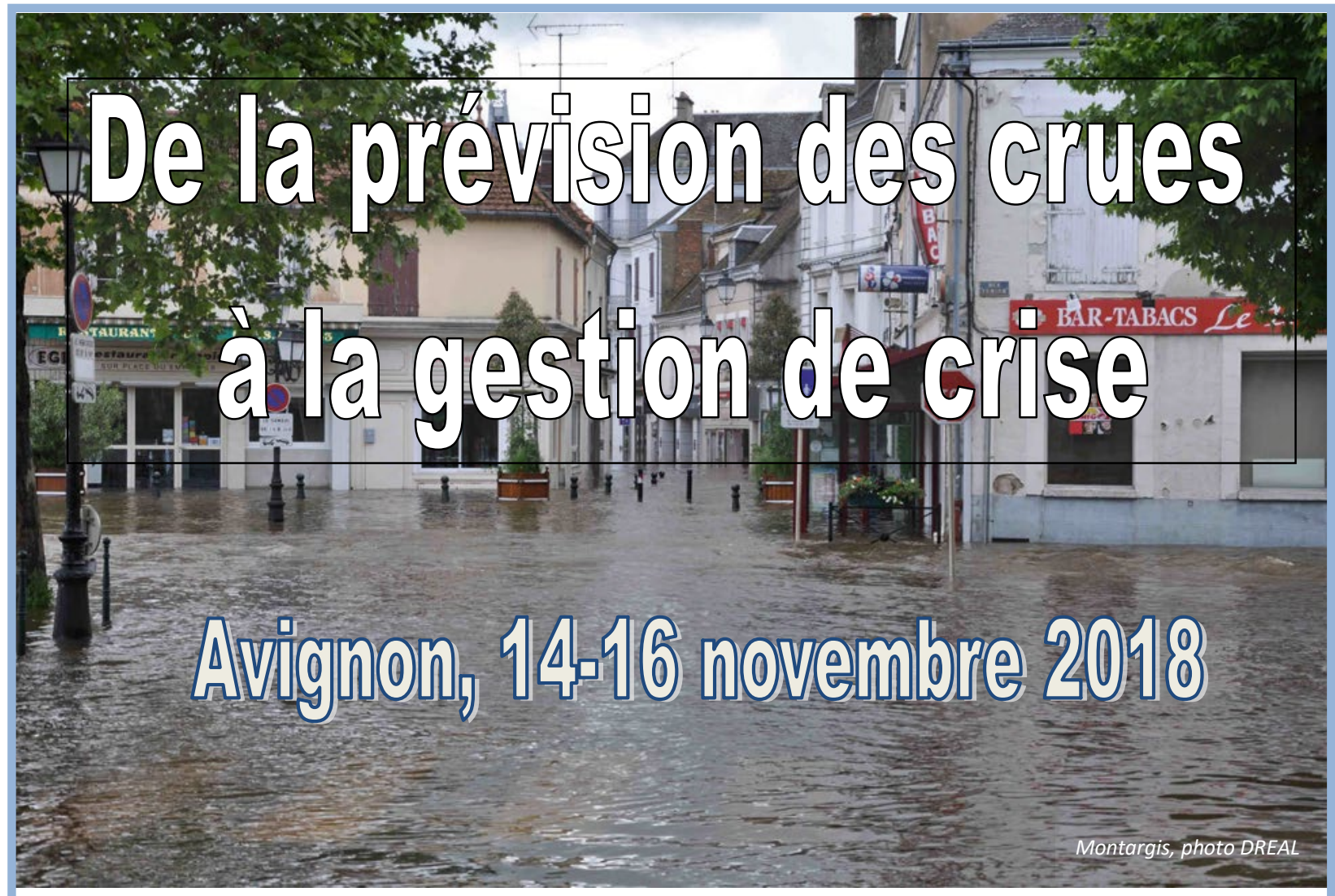

1/ Avancées scientifiques et opérationnelles sur la prévision des crues et des inondations 2/ De la préparation à la gestion de crise 